\title{
Una metodología de la formación para el audiovisual participativo europeo: Media Fellow
}

\section{Alma González Rodríguez y Marcelo Martínez Hermida} Universidade de Santiago de Compostela, España

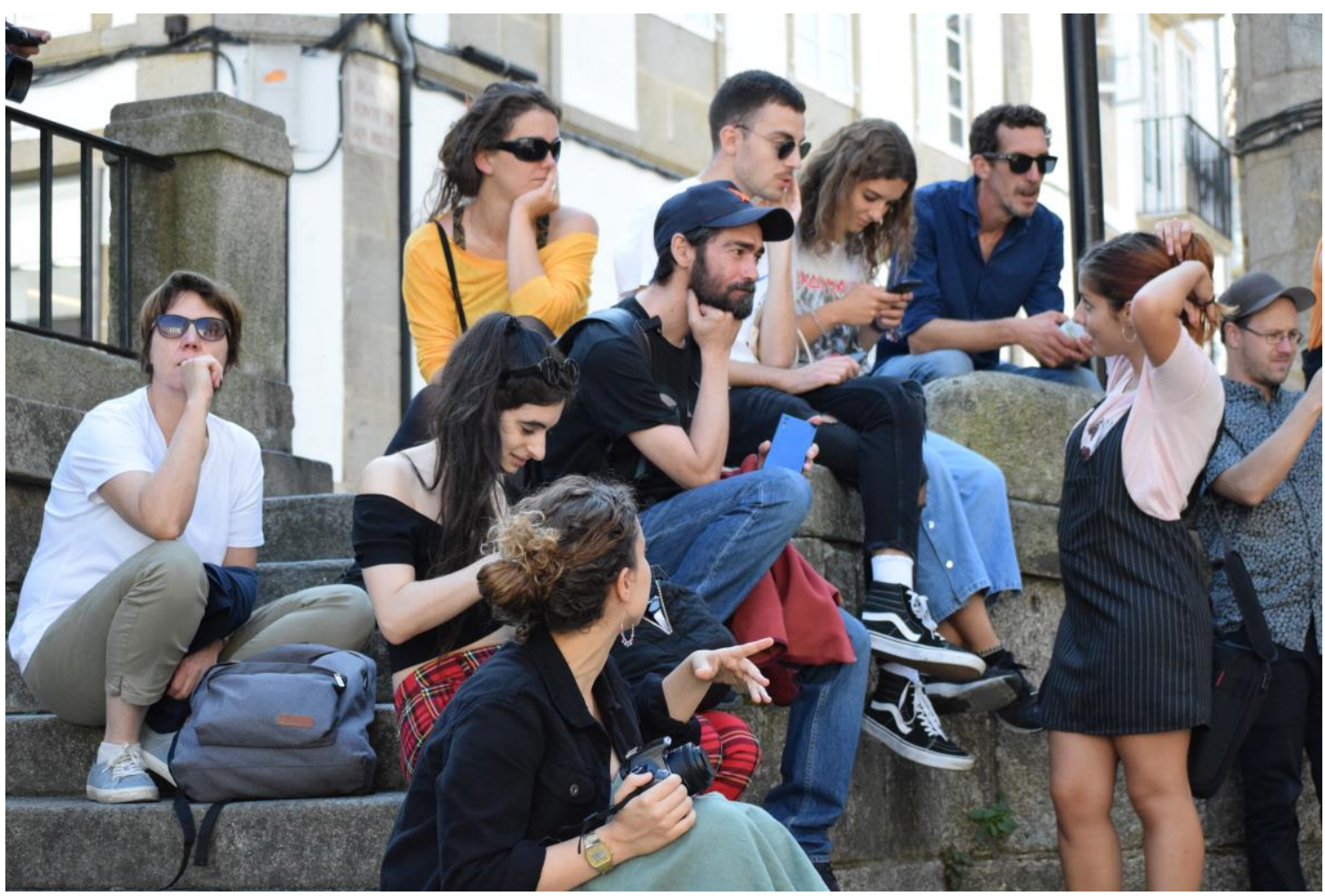

Participantes de la Ruta de la Comedia por Santiago de Compostela. Una actividad conjunta de Ios proyectos Media Fellowy Voluntarios no Audiovisual.

\section{NOTAS BIOGRÁFICAS}

Alma González Rodríguez es técnico de apoyo a la investigación en la USC. Graduada en Derecho y máster en Estudios Internacionales por la USC ha trabajado en el centro oficial de información dela Comisión Europea de la Deputación de A Coruña y ha sido voluntaria en ARCES (Italia).

Contacto:alma.gonzalez@usc.es

Marcelo Martínez Hermida es profesor de Comunicación Audiovisual y de Periodismo en la Facultade de Ciencias da Comunicación de la USC Licenciado en Periodismo por UAB y doctor en Ciencias de la Comunicación por UCM, es editor de la Revista Internacional de Comunicación y Desarrollo.

Contacto:marceloantonio.martinez@usc.es 


\section{INTRODUCIÓN}

A punto de que la Fédération de l'Audiovisuel Participatif francesa entregue la memoria final del proyecto Erasmus + KA2 Media Fellow, el grupo Cidadanía e Comunicación (CIDACOM) de USC repasa para RICD la experiencia y primeros avances de los resultados obtenidos sobre la formación en el audiovisual participativo europeo.

En este texto se adelantan algunos de los procesos y resultados obtenidos en el proyecto Media Fellow relativos a los métodos de la formación producida por entidades que actúan en el marco europeo del espacio asociativo, público y privado de diferentes países como Francia, Alemania y España; se describen y ponderan algunas de estas prácticas y se aporta una hoja de ruta para la formación en el audiovisual participativo europeo, a partir de las interacciones y aportaciones habidas durante este proyecto. El propósito de esta hoja de ruta es establecer un punto de partida para articular una formación diversa en audiovisual participativo desde los medios y organizaciones de este sector y para proponer una transferencia de técnicas que fortalezcan realmente las relaciones entre estas entidades y aquellas comunidades con las que comparten una producción de sentido rica y diversa que, a diferencia de los medios de masas, debe atender al común.

Hablar de audiovisual participativo plantea una metodología práctica que posiciona a la comunicación audiovisual en el mismo eje de la acción comunitaria, como herramienta colectiva para representarse a través de la participación y compartir procesos de transformación. A decir de Montero y Moreno (2020), dentro del campo académico, éste supone un objeto de estudio poco trabajado, aunque singular ya que "aporta a la academia muchas experiencias de dimensión práctica y genera conocimiento y reflexión, más desde la acción que desde el debate teórico.". Aunque singular, desde una mirada académica, tendremos que asumir que el objeto no es baladí si queremos considerar a la Comunicación como un campo dinámico y con cierta influencia en la articulación social y política, asociado a las urgencias comunitarias y ciudadanas para que obtengan por sí mismas un espacio donde compartir su voz, dispuestas a despojarse de los atributos que les impone el mainstream. También a la práctica justa de su derecho a comunicar, tan reivindicado actualmente por estos medios y grupos audiovisuales participativos en Europa, y todavía tan residual y desigual en muchas de las regulaciones de los países de la Unión Europea.

El ya largo compromiso de CIDACOM con la acción y el debate teórico sobre las comunidades y los espacios que construyen los medios $u$ otras instituciones de carácter público o social se ha reflejado -en concreto en el campo de la formación audiovisual comunitaria y participativa- en los estudios sobre la confluencia entre educomunicación y comunicación comunitaria (Mayugo Majó, 2015), sobre la formación audiovisual en los medios comunitarios españoles (Badía Deltell, 2018) o sobre el uso de los medios como instrumentos de cohesión comunitaria bajo distintos regímenes políticos (Amorín López, 2019); también en los debates sobre los asuntos que comparten distintas causas y perocupaciones ciudadanas como los sostenidos durante el proyecto CIDEC (Martínez Hemida, 2015) o en la creación y transferencia de herramientas docentes y de aportación social desde las academias como el Observatorio Cidadán de Comunicación (OCC) y su espejo latinoamericano Observatorio de Comunicación Ciudadana en UNEMI, Ecuador.

Desde el inicio del proyecto la participación de CIDACOM en Media Fellow se enfocó por tanto en recoger y valorar, durante los tiempos de intercambio de aprendizajes, el uso formativo de las prácticas y de las técnicas empleadas por los demás socios, incidiendo en su oportunidad y organización, en los recursos dispuestos para la formación, en la interacción entre formadores y aprendices, sobre los contenidos que se abordaron en los procesos de producción y el papel de los sujetos de la comunidad que intervienen en esos contenidos. También sobre las mejoras que proponen los operarios y voluntarios implicados en la experiencia formativa compartida.

Por otra parte, se facilitó a las entidades socias herramientas para que pudieran profundizar en los espacios de sus respectivos territorios y en las relaciones con sus comunidades; espacios y grupos de referencia que justifican el compartir de los socios en el audiovisual participativo. Instrumentos de diálogo que permiten indagar en los diferentes puntos de vista sobre aquellos asuntos relevantes para las comunidades o para desplegar una estrategia reticular sobre el territorio que permita documentar y proyectar grupos, recursos y temáticas de interés comunitario/participativo.

Para dejar constancia del proyecto y documentar algunas de las actividades de formación, CIDACOM fue realizando material audiovisual diverso: desde clips sobre los 
espacios del aprendizaje a entrevistas con los formadores o la grabación de las sesiones mantenidas en Santiago entre los socios, y de estos con otros agentes locales.

\section{TRANSFERENCIAS}

Tal como avanzaran Breuzin y Desset (2017) el objetivo del proyecto de cooperación en el que participó CIDACOM con la Fédération de L'Audiovisuel Participatif, Offener Kanal Magdeburg y Teleduca Educació i Comunicació se refería a la transferencia y a la adaptación de las técnicas y prácticas utilizadas por los distintos socios, con el fin de mejorar las competencias de sus operarios y voluntarios. Se pretendía, además, potenciar la capacidad de acción de las entidades participantes.

A propuesta de la Fédération el método para la organización de la formación se sustentó en el intercambio paritario de roles entre formadores y aprendices (es decir, estos operarios y voluntarios), según la formación se produjera en uno $\mathrm{u}$ otro territorio donde las entidades desarrollan habitualmente su labor audiovisual participativa y comunitaria. La movilidad de operarios y voluntarios entre los distintos espacios de producción audiovisual permitió que los anfitriones se encargaran de "acompañar" a los aprendices foráneos en las formas de producción y en las temáticas que normalmente abordan en el territorio, intercambiando papeles en Barcelona, Lille o Magdeburg, según la formación fuera en uno u otro de esos lugares elegidos para la experiencia de aprendizaje. Se utilizó como punto de partida la noción de compagnonnage, es decir, de tutelaje en la formación de los operarios y voluntadrios de los socios invitados a esas ciudades.

El seguimiento de la planificación de la formación y la organización del proyecto por parte de los responsables de las entidades se desarrolló en siete visitas: al mismo tiempo en que tuvo lugar la formación, durante la estancia en los tres lugares anteriormente ya mencionados, además de en Berlín (planificación del proyecto), Marsella (memoria final) y, en dos ocasiones, en Santiago de Compostela. En estas dos estancias intemedias se avanzó en herramientas de debate, análisis y conocimiento para la ponderación de la fomación y en instrumentos para fundamentar la reflexión y la reflexividad de sus procesos de producción y de participación en sus respectivos territorios.
La primera reunión en Santiago (abril, 2018) sirvió también a los socios para conocer de primera mano otras iniciativas de audiovisual participativo en Galicia (ACCOM, AGARESO, Airoa, Illa Bufarda, Proxecto Máscaras, Tintimán). En la segundda reunión (septiembre, 2018) se transfirieron aplicaciones de creación y mantenimiento de redes sociales humanas en los territorios, dispositivos de diálogo utilizados por CIDACOM o se implementaron herramientas de comunicación del proyecto. Los socios invitados a Santiago pudieron formar parte del proyecto Voluntarios no Audiovisual (Martínez Hermida y Ogando Des, 2018), financiado por el Concello de Santiago, o influir en la planificación de la experiencia de AV Coruña Común (Martínez Hemida y Ogando Des, 2019) patrocinado por la Deputación da Coruña; ambos auspiciados por CIDACOM. Es decir, Media Fellow facilitó la oportunidad para la interacción entre proyectos, y el intercambio de experiencias y material audiovisual realizado entre algunos de los grupos activos del audiovisual participativo gallego con sus colegas europeos.

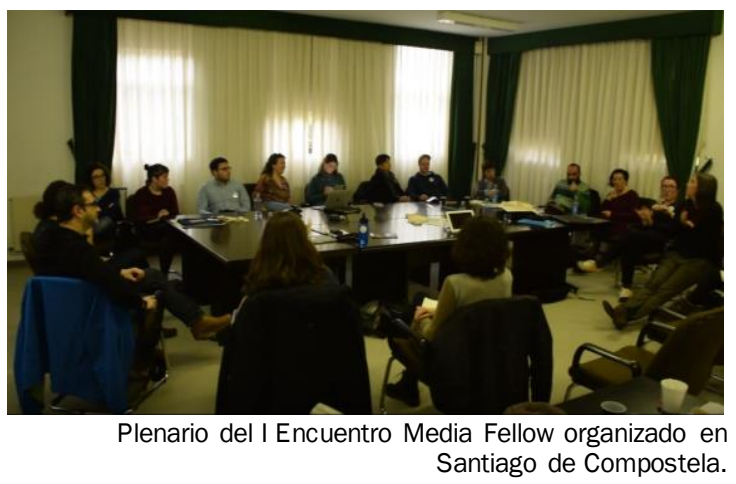

Algunos de estos socios, durante el desarroIlo de Media Fellow, practicaron posteriomente lo ensayado en Compostela en el marco de sus entidades y con grupos comunitarios de sus territorios. Herramientas como los círculos de lectura crítica - cuyo antecedente lejano podemos encontrar en los círculos de concientización de Paulo Freire (1973)- o una primera exploración de sus redes sociales humanas y su organización territorial como práctica para una cartografía social (Ares y Risler, 2013) que pueda facilitar la relación entidad-grupos del territorio, que permita el acceso de la comunidad al audiovisual y al diálogo sobre las temáticas y propuestas que preocupan a los grupos de ese territorio o sobre lo adecuado de las fomas de producción de su representación-participación. Se trataba de asegurar uno de los objetivos del proyecto, indicado al principio de esta reseña: potenciar la capacidad de acción no sólo entre entidades socias sino que ahora en sus 
territorios y con los grupos de la comunidad con quienes trabajan asiduamente. La producción en común de una epistemología de referencia a compartir en forma de glosario ha sido una de las herramientas que se ha considerado de mayor proyección para el ejercicio de aprendizajes futuros enfocados a operarios y voluntarios de estas entidades tan diversas. En este glosario se detallan conocimientos aquilatados en la experiencia audiovisual participativa europea o aquellos desarrollados de manera práctica durante las etapas de formación del propio proyecto. Se trata de un breve y útil muestrario, una caja de herramientas que siempre es necesario renovar, completar y, sobre todo, experimentar a fin de poder reflexionar y consolidar una mejor formación y un más intenso y provechoso aprendizaje. Entre la epistemología que surge desde el acervo propio y las experiencias de aprendizaje en común encontramos una escueta muestra de procesos y propuestas pedagógicas consolidadas históricamente en la tradición académica, del activismo social o desde la práctica operaria en el audiovisual participativo europeo como Learning by doing, Compagnonnage o Aktive Medienarbeiter, pero también instrumentos y técnicas de comunicación que, aunque novedosas en su propuesta, son herederas, por ejemplo, de una necesidad perentoria de que los medios participativos puedan reconocerse en el diálogo con las comunidades, como así potencia la práctica de los Círculos de Lectura.

\section{INSTRUMENTOS PARA LA RECOGIDA DE DATOS SOBRE LA FORMACIÓN}

Durante la inmersión en los lugares de aprendizaje estipulados previamente por los socios se practicó por parte del equipo de la Universidade de Santiago de Compostela (USC) una obsenación participante de la cual se extrajeron algunas impresiones in situ acerca de las buenas y malas prácticas en las rutinas de reflexión-producción propuestas por cada entidad, así como sobre la calidad de la fomación relacional y audiovisual ofrecida a operarios y voluntarios (incluyendo a los miembros del equipo de USC) o sobre las temáticas de proyección profesional y comunitaria que proporcionaron los formadores de cada entidad en cada aprendizaje situado. Formación en audiovisual participativo y capacidad en la recogida de datos sobre esa misma realidad de aprendizaje permitieron una contrastación sobre estas prácticas a los cuatro participantes de USC (dos técnicos de los servicios audiovisuales de la universidad y dos técnicos de apoyo a la investígación de esta misma institución). Se había abordado previamente con cada uno de los miembros del equipo un protocolo de actuación simple para esta observación. A su vuelta a Compostela se realizó un breve cuestionarioentrevista a los participantes de la USC sobre la obsenación practicada, a fin de cotejar con cierta perspectiva (una-dos semanas después) sus impresiones sobre dichas rutinas y temáticas.

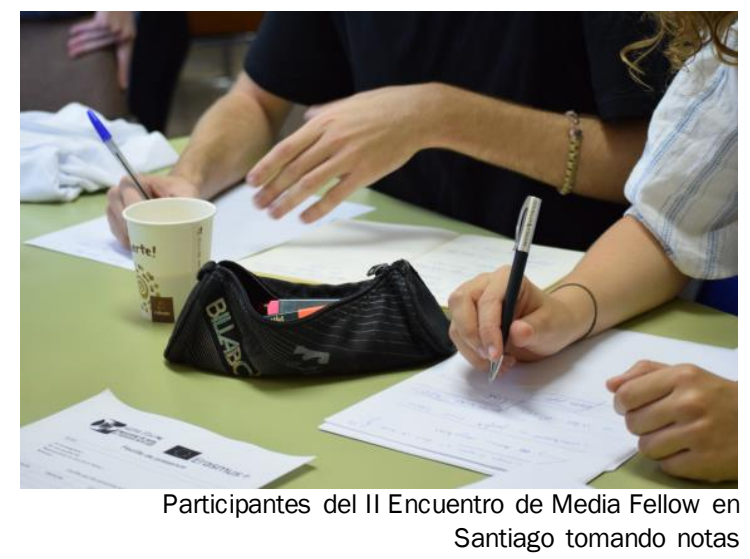

Las percepciones de los participantes de USC ayudaron en una lectura posterior que se llevó a cabo para comprobar las diferencias entre operarios y voluntarios, según lugar de procedencia y carácter de la entidad (público, privado, asociativo), con los datos obtenidos en otro cuestionario que cumplimentaran dichos operarios y voluntarios, participantes en cada uno de los tres lugares donde se participó la formación. Se repartió siempre este mismo cuestionario general a los asistentes. Se trataba de un documento compuesto por datos personales y sobre antecedentes formativos del participante (dado que cada entidad no hacía partícipes de la formación siempre a los mismos voluntarios, sino que alguno de ellos podía ser sustituido por otro dependiendo de la conveniencia de la entidad al movilizarse a uno u otro lugar), algunas preguntas con supuestos que tenían que evaluar sobre un baremo (contenidos, fuentes comunitarias, papel del formador,...) y una parte de preguntas abiertas en las que se les pedía proponer mejoras sobre la formación recibida en el lugar. En los recesos de la formación en las diversas ciudades se procedió a realizar unas breves entrevistas a los formadores, bien audiovisuales o bien ofreciéndoles en algún caso un guión que respondieron posteriormente por escrito. Se les preguntó por el proceso de aprendizaje llevado a cabo, la metodología seguida en el aula, las relaciones interculturales y profesionales que pudieran potenciar los conocimientos de ope- 
rarios y voluntarios, así como sobre el interés que creían hubieran despertado los contenidos, o la utilidad de tales conocimientos en la realidad de las prácticas habituales de las entidades.

Aunque exhaustivo se trató de un proceso de recogida de datos simple debido al escaso tiempo dedicado en los programas a la extracción de los datos, que se practicaron siempre durante intervalos disponibles, acomodados a la realización de la formación. A posteriori se completó alguna información para ratificar algunos resultados específicos obtenidos con búsquedas bibliográficas y trabajo de escritorio, preferentemente sobre documentos en línea de cada entidad. Esto ha sido importante, por ejemplo, en la apropiación y aplicación de conceptos y nociones sobre técnicas utilizadas por cada entidad o para comprobar la trayectoria y la proyección de cada entidad en los grupos humanos a los que se debe o con las instituciones que, en parte, financian sus actividades en sus territorios.

Los datos obtenidos de uno $u$ otro instrumento de recogida fueron cruzados y cotejados a partir de una matriz diseñada para evaluar el potencial de la formación en el audiovisual participativo, buscando constantes que, partiendo de la movilidad que facilitó el patrocinio europeo entre 2017 y 2020 , pudieran definir y mejorar este tipo de aprendizaje entre entidades de carácter diverso. El intercambio de prácticas y conocimientos sobre audiovisual participativo, el compartir operario sobre los procedimientos durante el proceso y en los resultados prácticos obtenidos de la producción, la transferencia de contenidos sobre las experiencias, propuestas y preocupaciones locales o la relación de cada una de estas entidades con los grupos humanos de su territorio y cómo ésta se refleja en la representación-participación de aquellos grupos han sido algunas de las cuestiones de referencia entresacadas de la comparación realizada mediante cuestionarios y entrevistas. Se obtuvieron, asimismo, consideraciones a tener en cuenta en la formación de intercambio sobre el carácter corporativo de cada entidad y de la aportación de cada grupo "nacional", sobre la estructura y organización o planificación de la formación, de la relevancia del aprendizaje en las actividades que cada entidad desenvuelve para la capacitación y para la captación de sus operarios, para su continuidad, para consolidar su referencia como activo social, cultural o de mercado y su consiguiente implantación en sus territorios.

\section{MARCOS Y CONSIDERACIONES PARAUNA METODOLOGÍA}

A la luz de la experiencia Media Fellow, y para pensar en una metodología del intercambio en el ámbito participativo-comunitario europeo, cabe explorar algunos marcos donde ubicar y planificar una formación que facilite la capacitación de los operarios y los voluntarios de aquellas entidades que se aventuraran a tal compromiso. Media Fellow ha resultado un proyecto donde se han probado e intercambiado, principalmente, procesos dialógicos entre operarios y técnicas de alfabetización audiovisual a las que muchos de estos trabajadores ya están acostumbrados por el carácter participativo-comunitario de sus entidades. Sin embargo, vistos los datos extraídos, la incorporación en la formación de voluntariado adscrito contractualmente a las entidades podría haber incidido en la obtención de una más clara resolución sobre el proceso de aprendizaje y en una más nítida expresión hacerca de la efectiva comprensión de los contenidos o, por ejemplo, en una valoración no tan homogénea sobre la idoneidad de las experiencias relacional e intercultural en el proyecto. Parece claro que la menor o mayor adscripción a la entidad definen distintos objetivos vitales y laborales, de participación y de capacitación de uno u otro grupo de formandos, y que ésta debiera ser tenida en cuenta a la hora de la selección de los participantes. Las técnicas de dinamización y la intensidad del aprendizaje, son dos cuestiones a tener muy en cuenta en la metodología y en la planificación de la formación, tanto si se trata de aunar a ambos agentes, o si solo se enfoca en uno de ellos.

Por otra parte, es necesario reparar en el reto que supone intercambiar modelos de emprendimiento que obedecen a propósitos diferentes aún en el mismo ámbito de actuación. Aunque pueda parecer enriquecedor el intercambio a la hora de ampliar la mirada u obtener procedimientos y nuevas técnicas que faciliten mejores procesos, para diseñar una metodología de formación de intercambio adecuada y efectova es muy relevante disponer y discutir previamente en el seno de cada entidad participante, sobre los materiales y experiencias que cada una de las entidades socias puedan facilitar a las demás y compartir esta información con formadores y participantes antes de emprender la formación. Los materiales preparatorios deberían incluir trayectoria y buenas prácticas sobre las relaciones de la entidad con grupos e instituciones de su entorno, agendas y tratamiento de los contenidos, propuestas y propósitos de for- 
mación. Disponer de una cartografía social contrastada o de un conocimiento sobre las técnicas relacionales y participativas que practica cada socio ayudará sin duda a generar una metodología de la formación más afinada que permita no sólo mostrar procedimientos, sino avanzar y mejorar en común. La transferencia de métodos de una entidad u otra en el intercambio debería trascender, según colegimos de la experiencia de Media Fellow, a la propia entidad y ser enfocada sobre propósitos comunes facilitando de esta manera menos distorsiones de carácter corporativo o identitario, profundizando y afianzando en la comunicación, en la tolerancia y en la riqueza de la interculturalidad.

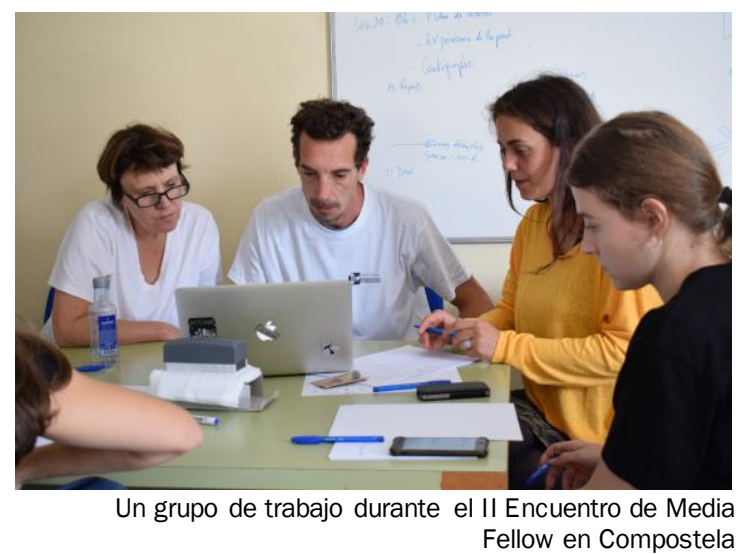

La formación en este tipo de entidades del audiovisual participativo suele ser una garantía para su continuidad social, laboral y de financiación. Es importante comprender que dicho intercambio se acoge en el marco político y regulatorio europeo donde el propósito es aunar esfuerzos y generar modelos comunes, que sean útiles como ejemplo de procesos educomunicativos y de educación sobre la comunidad, vitales para afianzar el diálogo territorial e intercultural y para viabilizar el acceso de los grupos locales a formas de representación propias y a su participación como inter-pares en el ejercicio pleno de la comunicación.

El proyecto Media Fellow dará paso a InMedia, otra iniciativa de las entidades socias que incorporará a otros compañeros de Polonia y Rumanía y que, sin duda, añadirá nuevos retos a la formación de intercambio. Tomando en consideración lo enunciado en este epígrafe aportamos a continuación una hoja de ruta con el ánimo de que sirva de orientación para construir en común una metodología, para ser discutida y asumida si acaso en el nuevo proyecto, posibilitando una formación de intercambio confiable y efectiva.

\section{DE MEDIA FELLOW A INMEDIA, PARA UN ESPACIO DEL AUDIOVISUAL PARTICIPATIVO EUROPEO}

Para el desarrollo del proyecto InMedia sería necesario facilitar una formación previa de sus operarios en el marco de cada entidad que participe lo siguiente:

a) información de experiencias y buenas prácticas de las entidades socias. Conviene que esta información, tipo informe de la experiencia y logros conseguidos, se acompañe de material audiovisual subtitulado y de procedimientos de lectura si fuera necesario para analizar lenguaje y tratamiento utilizados en la información de grupos vulnerables con los que se considere compartirvoz;

b) una cartografía de los grupos sociales e instituciones con los que se mantengan proyectos, propuestas e iniciativas locales, a fin de conocer la intensidad de actividades y la articulación de sus relaciones. En algunos casos es urgente recuperar las relaciones con el propio entorno, por ello es importante que en esta formación previa se abran espacios de interacción con los grupos sociales del territorio, de manera que los operarios actualicen su punto de vista acerca de propuestas y problemáticas con las que litigan estos grupos $y$, juntos, promuevan mejores representaciones y participaciones, mediaciones y soluciones, si es posible;

c) es importante que, revisado el material de las otras entidades socias en el proyecto, se elabore un glosario de técnicas que utilicen los operarios, las instituciones y los grupos sociales organizados del entorno, con el fin de repensar sobre sus procedimientos y proponer aquellas mejoras que se ajusten al interés del territorio, de los grupos sociales y en la mejora de la planificación y escritura audiovisual. En este glosario es importante la intervención de operarios, grupos e instituciones con las que se colabora normalmente. Esta epistemología inicial ayuda a identificar los métodos de la entidad y los métodos de la formación. Unos dirigidos a fundamentar sus decisiones (junto a la cartografía social practicada) y la transferencia de las mismas al conjunto de sus miembros, otros a preparar a sus operarios y orientar a sus voluntarios en el día a día desde su incomoración a la entidad. El intercambio de este material entre entidades socias en la formación internacional afinaría los argumentos y procesos de cooperación entre los operarios, reconociendo y avanzando en las técnicas y profundizando en su conocimiento mediante la práctica en común. 
Si es posible se debe estimar previamente entre las entidades socias si la formación de intercambio estará dirigida a operarios, o bien a operarios y voluntarios. En este aspecto es importante revisar conjuntamente las oportunidades que ofrece el marco del proyecto europeo al que se adscriben, respecto de los cupos estipulados para unos u otros formadoresformandos. Vista la experiencia de Media Fellow es importante que los voluntarios tengan un conocimiento previo sobre la formación de intercambio y lo que ésta les exigirá para completar el esfuerzo que implican tales procesos y marcos de relaciones.

Para InMedia, dado que el objetivo es incorporar técnicas de inclusión orientadas a los grupos sociales, dentro de los cupos de cada entidad sería muy importante hacer partícipes de la fomación de intercambio a miembros de instituciones y grupos sociales de cada territorio que habitualmente colaboran y comparten voz con la entidad socia. Ello ayudaría a comprender y a construir participación a través del audiovisual a estos grupos e instituciones, a valorar el esfuerzo de la entidad en facilitar su interacción con otros grupos e instituciones de otros territorios y a ayudar a interpretar, junto a los operarios de sus entidades, los proyectos y problemáticas que esos grupos proponen. Sería importante articular una metodología de fomación que acercara a los miembros de esos grupos sociales a la toma de decisiones en la construcción del relato audiovisual.

Algunos de los obstáculos en la fomación de intercambio que vienen a reflejar los cuestionarios de operarios y voluntarios que participaron en Media Fellow se refieren tanto a la falta de recursos de edición como a problemas de comunicación por la barrera de idiomas que dificultan la comprensión de los contenidos, sobre todo en las reuniones compartidas para transferir técnicas, propuestas y compartir el diálogo. Es importante mitigar esta situación y planificar con tiempo las dificultades incorporando a la ficha a la que nos referimos supra (ver en el punto "a" de este mismo epígrafe), la situación de equipamientos y la prevención de que asistan a la formación operarios y personas que faciliten sobrepasar tales barreras idiomáticas. De la misma manera es importante que el ejercicio de explicar in situ las metodologías y efectuar los controles del aprendizaje se incluyan en los tiempos de la formación de intercambio. En el primer caso la explicación de los avances en la metodología practicada no debe quedar en las reuniones de planificación. Las entidades socias deben de explicar a fomadores y fomandos cuáles son las prácticas que se deben realizar segundo las fases que se dispongan en el programa, antes de llevarlas a cabo. Los fomandos, por otra parte, deben conocer cuál es el objeto del control del aprendizaje.

Con respecto a la cartografía social que las entidades social deben operar en sus territorios se debe tener en cuenta qué grupos necesitan de su mediación comunicativa o con qué grupos se han establecido relaciones que motiven el interés de la entidad. Es preciso evaluar la red relacional de estos grupos y las facilidades y dificultades que tengan para promover el diálogo, así como su disponibilidad para establecer una cooperación efectiva. Sin embargo, en relación con el reto inclusivo que se propone de partida en InMedia, parece necesaria una mayor atención a aquellos grupos e individuos que no disponen ni de herramientas para el diálogo, ni de disponibilidad para participar. Sería importante localizar las rutas cotidianas de estos grupos sobre el mapa y acudir a los lugares para facilitar el encuentro. Estos grupos deben conocer y reconocer a la entidad como instrumento de inclusión donde expresar su representación, aquella desde la que reconocerse y ser reconocidos.

Para el proceso de selección y seguimiento de estos grupos sociales en el marco de la entidad es importante generar los siguientes mapas de contenido:

-Mapa de gentes. Esta agenda dentro de la comunidad buscará personas con las que la entidad haya tenido relación y considere de interés.

-Mapa de experiencias que refleje historia de vida del grupo, cuál es el discurso oficial sobre un determinado grupo, con quién tienen tensiones, problemas, etc.

-Mapa de lugares que recoja, entre otras cuestiones, dónde tienen lugar estos problemas o dónde se generan nuevas propuestas.

-Mapa de referencias o técnicas que utilizan estos grupos para incorporar al glosario.

Es importante conocer las redes del grupo con el que se trabaja: visión que poseen de su entorno, qué necesidades básicas tienen, etc. Eventualmente, se puede hacer uso de las redes que maneja el grupo en el territorio, obteniendo una red potencialmente más asentada y fundamentada para relacionar posibles mediaciones.

Conocer las competencias, ilusiones e intereses de los grupos sociales, lo que quieren 0 pueden compartir parece esencial a la hora de 
establecer el carácter de las relaciones y su confiabilidad en el momento de compartir su voz como sujetos, no como objetos de información. En este sentido debe de generarse un marco de retribución que reconozca la cooperación, tanto del grupo social que colabora, como de la entidad. Disponer de actividades en el que el audiovisual actúe como fedatario de acciones en común, entre operarios y comunidad, es importante para analizar en común el tratamiento de la información y mejorar las condiciones para consolidar una colaboración más confiable.

\section{REFERENCIAS BIBLIOGRÁFICAS}

- Amorín López, O. (2018). Espazos comunicativos como lugares de encontro social: dos teleclubs ás radios comunitarias en Galicia [tesis doctoral, Universidade de Santiago de Compostela, Santiago de Compostela]. Minerva. Repositorio Institucional da USC. http://hdl.handle.net/10347 /17415.

- Ares, P. y Risler, J. (2013). Manual de mapeo colectivo: recursos cartográficos críticos para procesos territoriales de creación colaborativa. Tinta Limón.

- Badía Deltell, C. (2019). Medios comunitarios en España: un mapa interactivo de la organización, formación y participación [trabajo fin de grado no publicado, Universidade de Santiago de Compostela].

- Breuzin, A.y Desset, T. (2018). Media Fellow, compartiendo prácticas por y para los medios participativos a escala europea, Revista Internacional de Comunicación y Desarrollo, 2(7), 131-134.

https://revistas.usc.gal/index.php/ricd/article/vie $\mathrm{w} / 4480$.

- Freire, P. (1973). ¿Extensión o Comunicación? La concientización en el medio rural. Siglo XXI- Ed. Tierra Nueva.

- Martínez Hermida, M. y Ogando Des, L.A. (2019). Av Coruña Común: breve cartografía do audiovisual participativo coruñés e galego. Revista Internacional de Comunicación y Desarrollo, 3(10), 159-165.

https://revistas.usc.gal/index.php/ricd/issue/vie $\mathrm{w} / 427$.

- Martínez Hermida, M. y Ogando Des, L.A. (2018). Voluntarios no Audiovisual. Revista Internacional de Comunicación y Desarrollo, 2(9), 156-160. https://revistas.usc.gal/index.php/ricd/article/vie w/5683.

- Martínez Hermida, M. (coord.) (2015). Cara a unha comunicación non excluínte. Caderno I. Proxecto CIDEC. Xunta de Galicia/Fondos FEDER UE.
- Mayugo Majó, C. (2015). La confluencia entre educomunicación y comunicación comunitaria en las prácticas de creación audiovisual participativa [tesis doctoral, Universidade de Santiago de Compostela]. Minerva. Repositorio Institucional da USC. http://hdl.handle.net/10347 /14611.

- Montero Sánchez D. y Moreno Domínguez, J. M. (2020). Explorando el campo de conocimiento del video participativo. Un recorrido por las principales aportaciones teórico-prácticas. Revista Internacional de Comunicación y Desarrollo, 3(11), 92-108. DOI: https://doi.org/10.15304/ricd.3.11.6345. 